\title{
An Improved Lag-Time Compensation Technique in Distributed Networked Control System Based on Smith Predictor
}

\author{
Ratish Kumar \\ Jaypee University of Information Technology, \\ Waknaghat - 173234, Solan, H.P., India \\ E-mail: ratishkumardhiman@gmail.com \\ Rajiv Kumar \\ Jaypee University of Information Technology, \\ Waknaghat - 173234, Solan, H.P., India \\ E-mail: rjv.ece@gmail.com \\ Madhav Ji Nigam \\ Jaypee University of Information Technology, \\ Waknaghat - 173234, Solan, H.P., India \\ E-mail: mkndnfec@gmail.com
}

Keywords: networked control system, smith predictor, delay compensation, kalman filter, markov approach, model estimation

Received: May 20, 2021

\begin{abstract}
The geographically distributed feedback control loops connected via communication network elucidate the core of networked control system (NCS), wherein different users spread all over the world can regulate, direct and command the components of control system located at distant location. The proficiency of NCS provides sceptre to its user and multifaceted assignments are successfully endowed through it. The ascendancy of NCS is lowered through inconspicuous delays which destabilize the system. The induction of delays in the network lead to the proscription of packet delivery, bandwidth utilization and stable system response. In this manuscript Smith predictor is modified using Markov approach and Kalman estimation algorithm. The scheme has been implemented by using Matlab/ Simulink software for delay compensation. Performance analysis shows the robustness of modified Smith-Predictor controller in comparison to classical Smith predictor controller and proportional-integral-derivative based controllers.
\end{abstract}

Povzetek: V prispevku je predstavljena nova tehnika za uravnavanje časovnih zaostankov v porazdeljenih nadzornih mrežnih sistemih.

\section{Introduction}

Rapid progress in communication technology has changed the perspectives of automation and control methodologies. Over the past few years; convergence of communication network and control system gave rise to the networked control system (NCS) as a novel approach of controlling the process or plants from a remote location. In NCS, to achieve the desired output, feedback control loops are spatially shared among the distributed actuators and sensors over the communication network [1]. In contrast to traditional point-to-point cable communication among various parts in a control system; the networked control system acquires less space, reduction in cabling complexity and power requirement that will lower the total installation and maintenance cost and hence, in turn will improve the reachability [2]. All these advantages have made the NCS popular among a wide range of applications including mobile robotics, traffic monitoring and control, aviation and satellite, defense and health care sector, etc. There are certain challenging issues such as time-variations in transmission, problem in network schedulability, network-induced delays, packet loss and bandwidth utilization etc., [3]. These days research community is taking keen interest towards these important problems related to NCS.

In networked control system, the randomness in network-induced delay is most noteworthy phenomenon that may occurs normally due to the limited channel bandwidth, network congestion, multipath transmissions or may be caused by a node waiting for the acknowledgement to transmit data over the channel. To maintain the high packing efficiency of data for multipacket transmission is also a challenging issue as all the scattered information is required to be packed [4]. During a transaction, due to the randomness, transmitted packets over the network are likely to be received in chaos that may lead to the packet dropout. In general, the data packet 
dropout means delay in receiving the data at the receiver node tends to infinite is mainly caused due to existence of

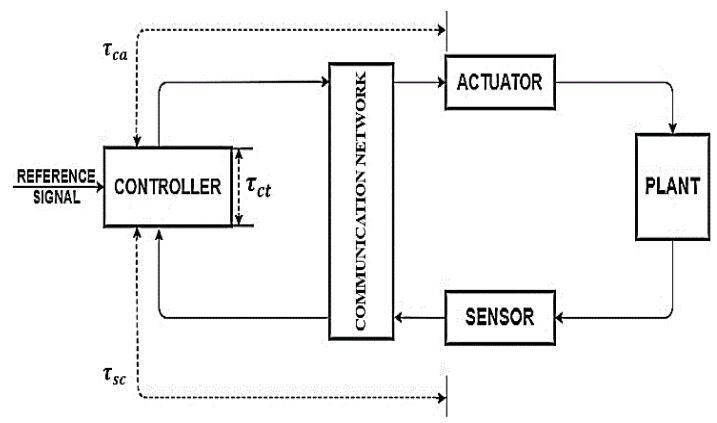

Figure 1: Major delays occurred in an NCS.

randomness in network-induced delays $[5,6]$. These network-induced delay affects the output response of the NCS which deprave the dynamic performance and also deteriorate the stabilized-control efficiency of the networked control system [7, 8]. Conventional control theory also finds it difficult to achieve at the solution of delay induced dynamical system, therefore, it is necessary to highlight the modeling of random network-induced delay while designing the NCS. Solution of the networkinduced random delay problem was sought by designing a networked predictive-controller [9]. Later on, networked predictive controller was advanced to facilitate the issues of constant and random network delays both in the forward and feedback channel [10, 11].

For NCS, a better system performance pretty much depends upon the timely reception of control signal by actuator and feedback signal truthfully measured by the sensor and its transference to the controller with infinitesimally small delay through communication network. Shown as in the Fig. 1, two types of networkinduced delays are associated with the networked control system.

The feedback delay or sensor-to-controller delay can be tracked in feedback channel between sensor and controller whereas, the feedforward delay or controller-toactuator delay can be traced between controller and actuator through the network. Figure 1 shows that controller and the plant are separated by a communication network which signifies the geographical reachability of the controlled operation but it also arises aforementioned delays.

In its intelligibly form the NCS can be represented in its state space model described as:

$$
\begin{aligned}
& x(k+1)=A x(k)+B u\left(k-\tau_{\text {total }}\right) \\
& y(k)=C x(k)
\end{aligned}
$$

where, $x \in R^{n}$ signifies system state vector, $u \in R^{m}$ represents the control input vector to the system, the matrices like A and B are system matrix and input matrix with their suitable dimensions and $\tau_{\text {total }}$ represents the total delay in the networked control system that majorly comprises of delay observed by controller due to sensor (s-c delay), delay spotted by actuator while transmitting packet from controller (c-a delay) and computational delay.

Hence, in NCS the prime concern is based upon the three delays as shown in Figure 1 are related as:

$$
\tau_{\text {total }}=\tau_{s c}+\tau_{c a}+\tau_{c t}
$$

the three types of delay mentioned in the equation (2), are sensor to controller delay $\tau_{s c}$, controller to actuator delay $\tau_{c a}$ and computational delay occurred due to the time taken by the controller to generate control signal for the actuator $\tau_{c t}$ in a networked control system.

To focus upon the networked induced delays the effect of computational delay $\tau_{c t}$ is ignored in this literature, the networked persuaded delays can be curtailed to reduce the total delay of the system. Most of the researchers have implemented proportional-integralderivative based Smith predictor controller to minimize the delay but did not mentioned about the uncertainty in the delay and estimation of the model for prediction [12, 13]. The main issue lies with the uncertainty observed in the network-induced delay which causes instability that are difficult to judge. So, an estimation of the randomness of delay and accuracy in the estimation of state model of the system is introduced in this paper. For which, Markov approach and Kalman estimation is considered in the literature in the feedback channel to estimate feedback delay in a Smith predictor controller [14, 15]. The remaining part of the manuscript is elaborated as follows: Section 2, explain the necessity of smith predictor, its limitations and the design of smith predictor for networked control system based on Markov approach and Kalman estimation. In Section 3, the simulation results are presented and compared to evaluate the best suited controller to bring improvement in the performance of the networked control system, followed by conclusion in Section 4.

\section{Development of Markov-Kalman based Smith predictor}

In the design of a NCS, time delay has been considered an important performance constraint. The sluggishness observed in the system response due to PI control action is improved with the incorporation of derivative action $[16,17]$. The robust performance for a wide range of operation fasten the Proportional-Integral-Derivative (PID) controllers for a variety of applications in the industry. Additional phase lag in the processes due to long

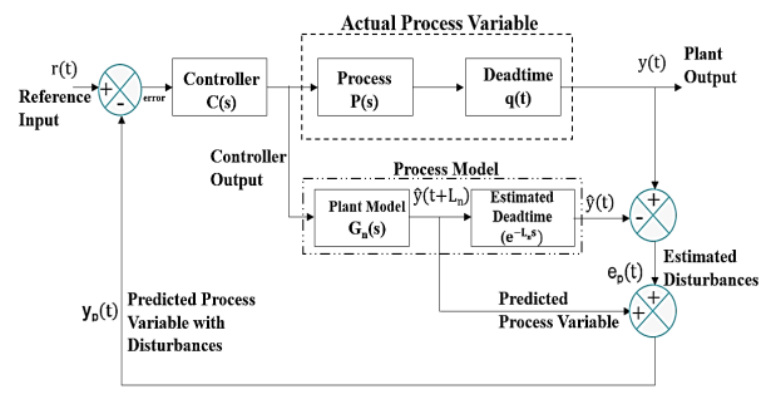

Figure 2: Classical Smith Predictor Model. 
time-delays are not easy to control efficiently with the use of PID controller and that may tend to destabilize the system. [18] defines how the Smith predictor can be compromised to compensate the delay time and stabilize the system. It has good set point response as it minimizes the delay characteristics of feedback loop.

To achieve the condition of zero error in the feedback channel it is required to get dead-time-free output $y_{p}(t)$ of the plant [19]. The prediction state in an SP is achieved by considering a process model without a time delay. The classical Smith predictor controller as shown in Figure 2, is comprise of two parts one is marked as primary controller C(s) which can be PID or any other higher order controller and second is predictor that consist of estimated parameters of the plant with transfer function represented as $G_{n}(s)$ and estimated dead time in the process mentioned as $\mathrm{e}^{-\mathrm{L}_{\mathrm{n}} \mathrm{s}}$ in Figure 2. Hence, the predictor module of the Smith predictor is given as $G_{n}(s) e^{-L_{n} s}$. It computes the open-loop prediction of system. The estimated deadtime can be obtained by taking the difference of output actual process variable and process model $[20,21]$.

Smith predictor is designed incoherence with Kalman filter and Markov approach as shown in Figure 3. The Markov approach is implemented to estimate the deadtime $\left(\mathrm{e}^{-\mathrm{L}_{\mathrm{n}} \mathrm{s}}\right)$ introduced in the process model to compare with the actual delayed feedback obtained from the plant output [22].

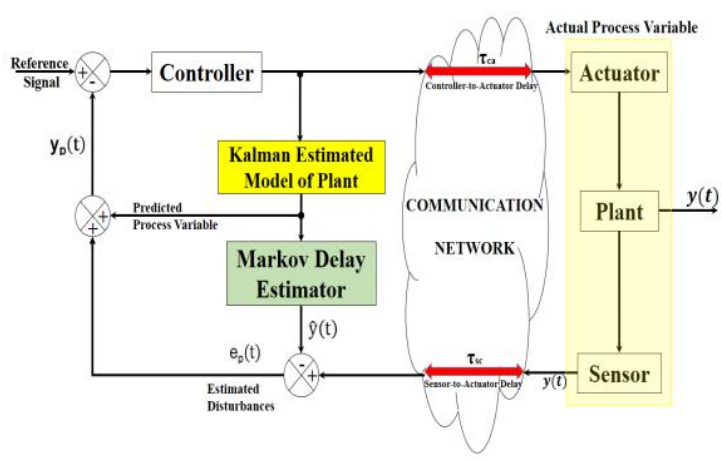

Figure 3: Markov and Kalman based Smith Predictor Controller.

This enable us to estimate the modified state space model of the plant given by equation 7 in section 2.1 . The Markov approach estimates the uncertain feedback delay and compares for estimated disturbance $e_{p}(t)$, which modify the disturbance variables for the predicted process. The algorithm developed for estimation of delay and packet dropout is mentioned in Table 1. Hence, the estimation of delay and packet drop modifies the state equation that are implemented in the Smith predictor controller [23].

\subsection{Random delay estimation using Markov model}

Markov analysis is considered as useful modeling and analysis methodology having vibrant applications in stochastic applications [24]. A state transition diagram can be used, that contain discrete state of the system and the

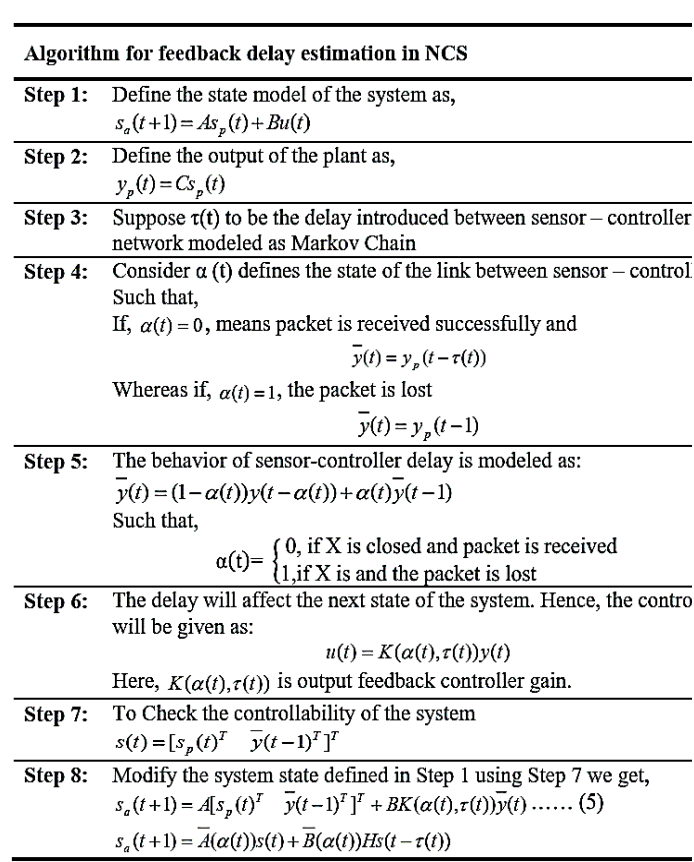

Table 1: Delay estimation algorithm using Markov approach.

future states can be examined by considering the rate of transition of states. Markov models define the consecutive representation of possible events between the two states shown in Figure 4, i.e. transitions within systems which, in the case of networked control system can be marked as the packet received or packet dropout lead to estimation of maximum delay. The Markov based delay estimation, counts the times and expected number of transitions of system between the possible states [25].

Markovian jump linear systems (MJLSs) are regarded as an elite group of hybrid systems with predictable operation approaches. The modelling of network-induced delays can be straightforwardly implemented through Markov models so as to foresee the behavior of delays in NCSs. This causes the probability distribution of transmission of data through the network that leads to the mode-independent control approach.

$$
\begin{aligned}
& s_{a}(t+1)=A s_{p}(t)+B u(t) \\
& y_{p}(t)=C s_{p}(t)
\end{aligned}
$$

$\mathrm{A}, \mathrm{B}, \mathrm{C}$ and D are known real constants with suitable proportions, $s_{p}(t) \in R^{n}, s_{a}(t+1), y_{p}(t)$ and $u(t)$ signifies present state of the system, future state of the system, plant output and control input to the plant respectively.

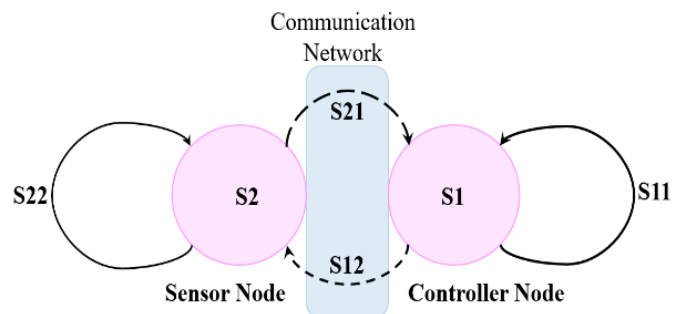

Figure 4: Markov model of induced delay in NCS. 
Since random time delay occurs across the network. The two types of delay include s-c and c-a called feedback and feedforward delays [26]. The feedback delays are more important as control inputs are function of feedback inputs. Delays lead to the packet dropout so it is important to cater them carefully so as to ensure the early prediction for the system state and input control signal [27]. The markov chain can be used to model the feedback in the state form and stabilization of the system can be obtained by optimizing the modeled state. Suppose $\tau(t)$ represent the delay of the feedback channel being modeled by markov chain [28,29]. As each component of a chain every other component similarly, while designing state of delay using markov chain delay measured (observed) in the one cycle will affect the future delay measurement, or we can say that previous state of delay will affect the current delay. So, the modeling of homogeneous markov chain for $\tau(t)$ can take values.

$$
\bar{X}_{2}=\left\{0,1,2 \ldots, x_{2}\right\}
$$

$\mathrm{X}$ denotes network switches between s-c, $\alpha(t)$ denotes the state s and $\alpha(t)=[0,1]$

When $\mathrm{X}$ is in $\alpha(t)=0$, depicts the successful reception of data packet and

$$
\bar{y}(t)=y_{p}(t-\tau(t))
$$

Whereas if $\mathrm{X}$ is in the state $\alpha(t)=1$ the packet is lost

$$
\bar{y}(t)=y_{p}(t-1)
$$

The modelling of sensor-to-controller delay can be represented as,

$$
\bar{y}(t)=(1-\alpha(t)) y(t-\alpha(t))+\alpha(t) \bar{y}(t-1)
$$

$$
\alpha(t)
$$

where,

$=\left\{\begin{array}{l}0, \text { if packet received shows } X \text { is closed } \\ 1, \text { if packet is lost thus the packet is lost }\end{array}\right.$

Now, input to mode dependent output feedback controller presented as:

$$
\mathrm{u}(\mathrm{t})=\mathrm{K}(\alpha(\mathrm{t}), \tau(\mathrm{t})) \mathrm{y}(\mathrm{t})
$$

Here, $K(\alpha(t), \tau(t))$ is output feedback controller gain.

Suppose, $s(t)=\left[\begin{array}{ll}s_{p}(t)^{T} & \bar{y}(t-1)^{T}\end{array}\right]^{T}$ is augmented state vector. For control vector (6) the closed loop system of (1) becomes,

$$
\begin{aligned}
& s_{a}(t+1)=A\left[s_{p}(t)^{T} \quad \bar{y}(t-1)^{T}\right]^{T}+B K(\alpha(t), \tau(t)) \bar{y}(t) \\
& s_{a}(t+1)=\bar{A}(\alpha(t)) s(t)+\bar{B}(\alpha(t)) H s(t-\tau(t)) \\
& s(t)=\varphi(t) \\
& t=-\tau_{\max },-\tau_{\max }+1, \ldots . .0
\end{aligned}
$$

Here,

$$
\begin{aligned}
& \bar{A}(\alpha(t))=\left[\begin{array}{cc}
A \alpha(t) & B K(\alpha(t), \tau(t) \\
0 & \alpha(t) I
\end{array}\right] \\
& \bar{B}(\alpha(t))=\left[\begin{array}{c}
(1-\alpha(t)) B K(\alpha(t), \tau(t)) C \\
(1-\alpha(t)) C
\end{array}\right] \\
& H=\left[\begin{array}{ll}
I & 0
\end{array}\right], \tau_{\max }=\max (\tau(t)) \text { and } \varphi(t) \text { is initial condition }
\end{aligned}
$$
of $x(t)$.

In the system (7), $\{\alpha(t), t \in \mathrm{Z}\}$ and $\{\tau(t), t \in \mathrm{Z}\}$ are two independent discrete time homogeneous markov chains

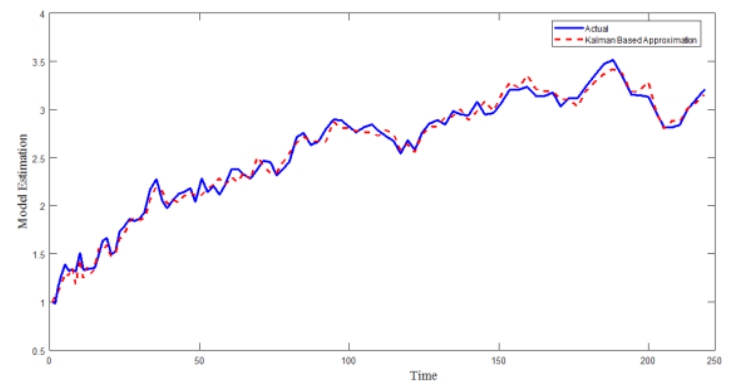

Figure 5: Model estimation of plant.

taking values in a finite set $\bar{X}=\{0,1\}$ and $\overline{X_{2}}=\left\{0,1,2, \ldots, x_{2}\right\}$ with transition probabilities:

$$
\begin{aligned}
& P_{r}\{\alpha(t+1)=j \mid \alpha(t)=i\}=\Pi_{i j} \text { from } \mathrm{i} \text { to } \mathrm{j} . \\
& \Pi_{i}=P_{r}\left(\Pi_{0}=i\right) \\
& P_{r}\{\tau(t+1)=n \mid \tau(t)=m\}=\lambda_{m n} \text { from } \mathrm{m} \text { to } \mathrm{n} .
\end{aligned}
$$$$
\text { where, } \Pi_{i j} \geq 0 \text { and } \lambda_{m n} \geq 0 \text { for all } i, j \in Z_{1}, Z_{2}
$$$$
\sum_{j=0}^{1} \Pi_{i j}=1 \text { and } \sum_{n=0}^{s_{2}} \lambda_{m n}=1
$$

For $\alpha(t)=i, i \in \overline{X_{1}}$ and $\alpha(t)$ in mode $\mathrm{i}=0$ and $\mathrm{i}=1$ the $\alpha(t)$ in (7) take value $\alpha(t)=0$ and $\alpha(t)=1$ respectively. $\bar{A}(\alpha(t))$ and $\bar{B}(\alpha(t))$ are known constant matrices of appropriate dimensions.

The closed loop in equation (7) is a markov jump linear system with two markov chains that describe the behavior of the system for s-c time delays and packet dropouts respectively. This enables to analyze and synthesize such NCS by applying markov jump linear system and help us to estimate the modified state space model of the plant [30,31]. The Markov approach estimates the uncertain feedback delay and compares for estimated disturbance $e_{p}(t)$, which modify the disturbance variables for the predicted process.

\section{Results and discussion}

To illustrate the effectiveness of the proposed modified smith predictor it is compared with different controllers such as PI, PD, PID and Smith predictor. A comparison is drawn in Table 2. The modified smith predictor gives $34.16 \%$ improvement in settling time as compared to conventional Smith predictor, which shows that modified Smith predictor is an effective deadtime compensator for a stable process with long time-delays. The delay induced is modeled using Markov approach as shown in Figure 6, representing the transfer function of the plant model is

$P(s)=\frac{127}{s^{3}+15 s^{2}+83 s+101.3}$

The stability problem can be solved by decreasing the controller gain. However, in this case the response obtained for smith predictor is very sluggish about $519.024 / \mathrm{ksec}$ which is further improved by modified Smith predictor $71.337 / \mathrm{psec}$, a faster slew rate improves the bandwidth of the system and hence reduce the time delay and probability of packet dropout. The model estimation for the prediction of ideal state of the plant is 
performed through Kalman estimation algorithm as shown in Figure 5.

PID controllers are the workforce of the industry but with the high demand of precision they are becoming sluggish the response of the PI, PD and PID can be observed from the Figure 7(a). The slew rate of the simulated controllers for networked control system can be compared from Table 2. Where lowest slew rate is
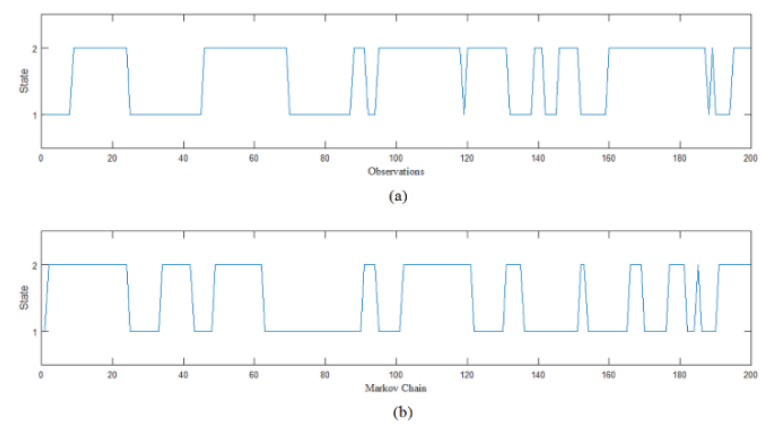

Figure 7: (a) Markov model-based observation (b) Sequence of Markov Chain.

\begin{tabular}{|l|l|l|l|}
\hline \multirow{2}{*}{ Parameters } & \multicolumn{3}{|c|}{ Type of Controllers } \\
\cline { 2 - 4 } & PID & $\begin{array}{l}\text { Smith } \\
\text { Predictor }\end{array}$ & $\begin{array}{l}\text { Proposed } \\
\text { Modified Smith } \\
\text { Predictor }\end{array}$ \\
\hline $\begin{array}{l}\text { Rise Time (units } \\
\text { of time) }\end{array}$ & $0.572 \mathrm{sec}$ & $0.0015 \mathrm{sec}$ & $0.00012 \mathrm{sec}$ \\
\hline $\begin{array}{l}\text { Slew Rate (per } \\
\text { units of time) }\end{array}$ & $\begin{array}{l}1.398 \\
/ \mathrm{sec}\end{array}$ & $\begin{array}{l}519.024 \\
/ \mathrm{ksec}\end{array}$ & $71.337 / \mathrm{psec}$ \\
\hline Preshoot (in \%) & 0.521 & 0.505 & 0.505 \\
\hline Overshoot (in \%) & 3.646 & 0.501 & 0.505 \\
\hline $\begin{array}{l}\text { Undershoot (in } \\
\text { \%) }\end{array}$ & 1.996 & 1.997 & -0.505 \\
\hline $\begin{array}{l}\text { Settling Time ( in } \\
\text { Sec) }\end{array}$ & $0.39 \mathrm{sec}$ & $0.48 \mathrm{sec}$ & $0.316 \mathrm{sec}$ \\
\hline
\end{tabular}

Table 2: Comparison of performance parameters of proposed modified Smith predictor controller with conventional controllers.

provided by PI controllers and higher slew rates in modified Smith predictor structure can be observed which offers a robust improvement in performance of closed loop over traditional controllers for stable processes.

In our design we have used values, $\mathrm{P}=2.0, \mathrm{I}=3.4$ and $\mathrm{D}=0.1$ for a continuous-time PID controller to design the modified Smith predictor for networked control system with state space model of stepper motor represented as:

$\mathrm{A}=\left[\begin{array}{ccc}-11 & -91 & -108.3 \\ 1 & 0 & 0 \\ 0 & 1 & 0\end{array}\right], \quad \mathrm{B}=\left[\begin{array}{l}1 \\ 0 \\ 0\end{array}\right], \quad \mathrm{C}=\left[\begin{array}{lll}0 & 0 & 107\end{array}\right]$ and $\mathrm{D}=[0]$

The NCS has broadly two types of applications, one is time sensitive i.e., time critical and second is time insensitive applications also termed as time non-critical applications.

\section{Conclusion}
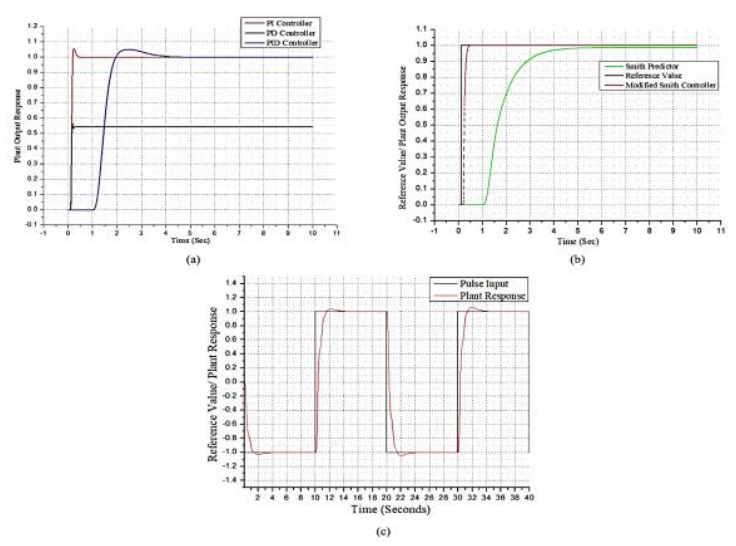

Figure 6: (a) Comparison of Plant Response using PI, PD and PID Controllers, (b) Comparative response of proposed modified predictive controller and conventional Smith predictor, (c) Response of proposed modified predictive controller to pulse input is evident from the Figure 7(b) that the modified Smith predictor have rise-time of $1.2 \times 10-6 \mathrm{sec}$ which is suitable for time sensitive applications such as medical and defense applications. From Figure 7(c) the impulse response of the modified Smith predictor can be observed with rise time of $0.78 \mathrm{sec}$ and overshoot of $1.9 \%$.

In case of NCS, network-induced delays are found very critical because these delays severely affect the performance of system. In order of compensate these delays, this paper proposes a modified Smith predictor controller. Proposed predictive controller adopts the Markov modeling for estimation of random delays and Kalman predictive approach is implemented to estimate the plant model of the Smith predictor model. Therefore, based upon these modifications proposed predictive controller is able to compensate effectively the delays with uncertainty. In this paper simulations with help of Matlab/ Simulink have been performed for various delay compensation schemes. Results ensure that the proposed modified Smith predictive controller scheme have a noteworthy improvement over Smith Predictor (without Markov) and other conventional controllers in terms of rise-time, slew rate, overshoot and settling time. Hence it can be précised that proposed modified predictor is appropriate for time critical applications where delay with randomness influences the performance of networked control system.

\section{Acknowledgement}

The authors are also sincerely thankful to the anonymous reviewers for their treasured comments and recommendations in order to improve the quality of this paper. We are also really thankful to Department of Electronics and Communication Engineering, Jaypee University of Information Technology for the support during the Pandemic (Covid-19) situation. 


\section{References}

[1] T. C. Yang, "Networked control system a brief survey," IEE Proceedings - Control Theory and Applications, vol. 153(4), pp. 403-412, 2006. https://doi.org/10.1049/ip-cta:20050178

[2] J. Wu and T. Chen, "Design of Networked Control Systems with Packet Dropouts," IEEE transactions on Automatic Control, vol. 52 (7), pp. 1314-1319, 2007. https://doi.org/10.1109/TAC.2007.900839

[3] S. Bhuyan, B. Subudhi and S. Ghosh, "Networked control system: uncertainty modeling and stabilization," Procedia Engineering, vol. 38, pp. 3662-3667,

2012. https://doi.org/10.1016/j.proeng.2012.06.422

[4] R. A. Gupta and Mo-Yuen Chow, "Networked Control System: Overview and Research Trends," IEEE Transactions on Industrial Electronics, vol. 57(7), pp. 2527-2535, 2010. https://doi.org/ 10.1109/TIE.2009.2035462

[5] H. Gao, T. Chen and J. Lam, "A new delay system approach to network-based control," Automatica, vol. 44 (1), pp. 39-52, 2008. https://doi.org/10.1016/j.automatica.2007.04.020

[6] J. Baillieul and P. J. Antsaklis, "Control and communication challenges in networked real-time systems," Proceedings of the IEEE, vol. 95 (1), pp. 928, 2007. https://doi.org/10.1109/JPROC.2006.887290

[7] A. Ingimundarson and T. Hägglund, "Robust tuning procedures of dead-time compensating controllers," Control Engineering Practice, vol. 9(11), pp. 11951208, 2001. https://doi.org/10.1016/S0967-0661(01)00065-X

[8] A. Sharma, P. K. Singh, A. Sharma and R. Kumar, " An efficient architecture for the accurate detection and monitoring of an event through the sky," Computer Communications, vol. 148, pp. 115-128, 2019. https://doi.org/10.1016/j.comcom.2019.09.009

[9] Q. C. Zhong, "Unified Smith predictor for dead-time systems," IFAC Proceedings Volumes, vol. 36 (19), pp. 83-86, 2003. https://doi.org/10.1016/S14746670(17)33306-2

[10]Hui Wang, "Research on Data Transmission Optimization of Communication Network Based on Reliability Analysis," Informatica, vol. 44, 361-366, 2020. https://doi.org/10.31449/inf.v44i3.3280

[11] W. Jing, Z. Liqian and C. Tongwen, "An MPC approach to networked control design," in 2007 Chinese Control Conference, IEEE, 2007, pp. 10-14. https://doi.org/10.1109/CHICC.2006.4346915.

[12]A. Sharma and R. Kumar, "Service-level agreement - energy cooperative quickest ambulance routing for critical healthcare services," Arabian Journal for Science and Engineering, vol. 3848, no. 4, p. 3831, 2019. https://doi.org/10.1007/s13369-0183687-z

[13] M. Gamal, N. Sadek, R. M. Rizk and A.-e. A. K., "Delay compensation using Smith predictor for wireless network control system," Alexandria
Engineering Journal, vol. 55, no. 2, pp. 1421-1428, 2016. https://doi.org/10.1016/j.aej.2016.04.005

[14] Grimholt and S. Skogestad, "Optimal PID control on first order plus time delay systems \& verification of SIMC rules," IFAC Proceedings Volumes, vol. 46 (32), pp. 265-270, 2013. https://doi.org/10.3182/20131218-3-IN-2045.00122

[15] Hai-Anh Tran, Duc Tran, Linh-Giang Nguyen, QuocTrung Ha, Van Tong and Abdelhamid Mellouk, "SHIOT: A Novel SDN-based Framework for the Heterogeneous Internet of Things," Informatica, vol. 42, pp. 313-323, 2018. https://doi.org/10.31449/inf.v42i3.2245

[16] R. E. Kalman, "A New Approach to Linear Filtering and Prediction Problems," Transactions of the ASME-Journal of Basic Engineering, vol. 82, no. D, pp. 35-45, 1960. https://doi.org/10.1115/1.3662552

[17] A. Sharma and R. Kumar, "Performance comparison and detailed study of AODV, DSDV, DSR, TORA and OLSR routing protocols in ad hoc networks," in 2016 Fourth International Conference on Parallel, Distributed and Grid Computing (PDGC), Waknaghat, India, 2016. https://doi.org/10.1109/PDGC.2016.7913218

[18]F. S. Oliveria, F. O. Souza and R. M. Palhares, "PID tuning for time-varying delay systems based on modified smith predictor," IFAC-PapersOnLine, vol. 50 (1), pp. 1269-1274, 2017. https://doi.org/10.1016/j.ifacol.2017.08.130

[19] G. Rathee, A. Sharma, R. Kumar, F. Ahmad and R. Iqbal, "A trust management scheme to secure mobile information centric networks," Computer Communications, vol. 151, pp. 66-75, 2020. https://doi.org/10.1016/j.comcom.2019.12.024.

[20] F. Peterle, M. Rampazzo and A. Beghi, "Control of Second Order Processes with Dead Time: the Predictive PID Solutions," IFAC-PapersOnLine, vol. 51 (4), pp. 793-798, 2018. https://doi.org/10.1016/j.ifacol.2018.06.183

[21] Mouadh Bali, Abdelkamel Tari, Abdallah Almutawakel and Okba Kazar, "Smart Design for Resources Allocation in IoT Application Service Based on Multi-agent System and DCSP," Informatica, vol. 44, pp. 373-386, 2020. https://doi.org/10.31449/inf.v44i3.2962

[22] Y. Liu, L.S. Hu and P. Shi, "A novel approach on stabilization for linear systems with time-varying input delay," vol. 218(10), pp. 5937-5947, 2012. https://doi.org/10.1016/j.amc.2011.11.056

[23]P. Batista and F. G. Jota, "Performance improvement of an NCS closed over the internet with an adaptive Smith Predictor," Control Engineering Practice, vol. 71, pp. 34-43, 2018. https://doi.org/10.1016/j.conengprac.2017.10.006

[24]H. Gao, X. Meng and T. Chen, "Stabilization of Networked Control Systems with a New Delay Characterization," IEEE Transactions on Automatic Control, vol. 53 (9), pp. 214-2148, 2008. https://doi.org/10.1109/TAC.2008.930190

[25] Varshney, M. Bhushan and S. C. Patwardhan, "State and parameter estimation using extended Kitanidis 
Kalman filter," Journal of Process Control, vol. 76, pp. 98-111, 2019.

https://doi.org/10.1016/j.jprocont.2018.11.007

[26] Y. Ishido, K. Takaba and D. E. Quevedo, "Stability analysis of networked control systems subject to packet-dropouts and finite-level quantization," Systems \& Control Letters, vol. 60 (5), pp. 325-332, 2011. https://doi.org/10.1016/j.sysconle.2011.02.008

[27] O. L. Costa and M. D. Fragoso, "Stability results for discrete-time linear systems with Markovian jumping parameters," Journal of mathematical analysis and applications, vol. 179 (1), pp. 154-178, 1993. https://doi.org/10.1006/jmaa.1993.1341

[28]Zelinka and B. Amadei, "A Systems Approach for Modeling Interactions among the Sustainable Development Goals Part 2: System Dynamics," International Journal of System Dynamics Applications, vol. 8, no. 1, pp. 41-59, 2019. https://doi.org/10.4018/IJSDA.2019010103

[29]Leila Boucerredj and NasrEddine Debbache, "Qualitative and Quantitative Optimization for Dependability Analysis," Informatica, vol. 42, pp. 493-450, 2018.

https://doi.org/10.31449/inf.v42i3.1580

[30] J. Adam, H. A. Latchman and O. D. Crisalle, "Robustness of the Smith predictor with respect to uncertainty in the time-delay parameter," in Proceedings of the 2000 American Control Conference. ACC (IEEE Cat. No. 00CH36334), IEEE, 2000, pp. 1452-1457.

https://doi.org/10.1109/ACC.2000.876742

[31]Zhai, D. Xue, S. Li and G. Wang, "Stabilization of discrete-time Markovian jump systems via controllers with partially mode-dependent characterization," Mathematical Problems in Engineering, vol. 2013, pp. 1-9, 2013. https://doi.org/10.1155/2013/936375 
